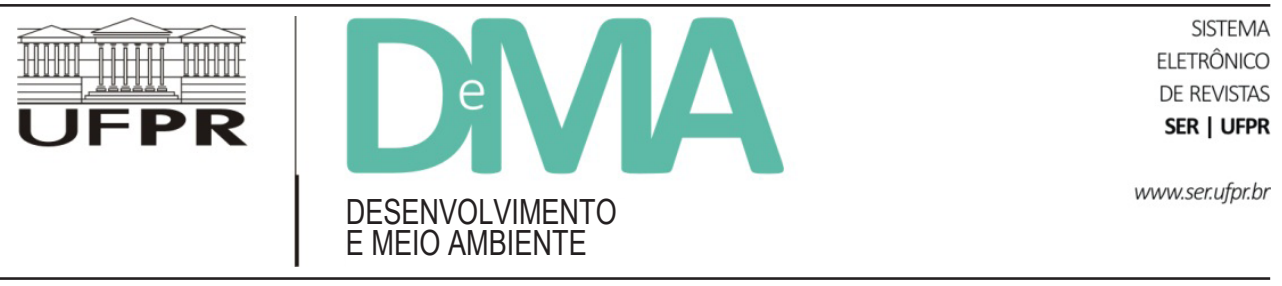

\title{
O uso da Avaliação Ambiental Estratégica nas decisões sobre cultivares transgênicos no Brasil
}

\section{The Strategic Environmental Assessment in Decision Making about Transgenic Crops in Brazil}

\author{
Denise Gallo PIZELLA ${ }^{1 *}$, Marcelo Pereira de SOUZA ${ }^{2}$ \\ ${ }^{1}$ Universidade Estadual Paulista "Júlio de Mesquita Filho" (UNESP), Ilha Solteira, SP, Brasil. \\ ${ }^{2}$ Departamento de Educação, Informação e Comunicação, Faculdade de Filosofia, Ciências e Letras de Ribeirão Preto, Universidade de São \\ Paulo (USP), Ribeirão Preto, SP, Brasil. \\ *E-mail de contato: denise@bio.feis.unesp.br
}

Artigo recebido em 20 de março de 2014, versão final aceita em 19 de fevereiro de 2015.

RESUMO: A liberação ambiental de cultivares transgênicos é polêmica, devido às incertezas quanto aos potenciais impactos ambientais e socioeconômicos que pode suscitar. De modo a regular as deliberações sobre o uso de organismos geneticamente modificados (OGMs), mecanismos regulatórios que se propõem a prever tais impactos foram criados em diversas nações, sendo a análise de risco (AR) o instrumento de estudo ambiental normalmente empregado nos processos decisórios. No entanto, há contestações sobre seu uso como única ferramenta de análise ambiental destes OGMs, já que não possibilita a avaliação dos impactos cumulativos, indiretos, de longo prazo e dos interesses das nações delineados em suas políticas, planos e programas (PPPs). Um instrumento proposto por alguns autores passível de abarcar tais considerações é a Avaliação Ambiental Estratégica (AAE), que busca inserir a variável ambiental durante as fases de planejamento que resultam na elaboração de PPPs. Este trabalho teve como objetivo identificar as contribuições da utilização da AAE para subsidiar os processos decisórios que envolvem a liberação ambiental de cultivares transgênicos no Brasil. Tendo em vista que esta atividade é estratégica para a agricultura brasileira, buscou-se analisá-la segundo os parâmetros que condicionam as boas práticas de AAE. Observou-se, deste modo, que a AAE poderá contribuir para a tomada de decisões no tocante à liberação comercial destas cultivares, em função de seus princípios de transparência, envolvimento social, planejamento ambiental, abrangência de amplos recortes territoriais, avaliação de impactos cumulativos e de longo prazo e monitoramento ambiental contínuo. Deste modo, recomendou-se a utilização da AAE no planejamento ambiental envolvendo liberações de cultivares transgênicos no Brasil.

Palavras-chave: OGM; biossegurança; planejamento ambiental.

ABSTRACT: The environmental use of transgenic crops is controversial, due to the uncertainties regarding the potential environmental, social and economic impacts that may arise. In order to regulate the decisions on the use of genetically modified organisms (GMOs), regulatory mechanisms that aim to predict such impacts were im- 
plemented in different countries, the risk analysis (RA) being the environmental study method normally used for making such decisions. However, there are objections as to its use as the unique tool for the environmental analysis of GMOs, since it does not allow the assessment of cumulative, indirect and long-term impacts and interests of countries as outlined on their policies, plans and programs (PPPs). One tool proposed by some authors likely to encompass such considerations is the Strategic Environmental Assessment (SEA), which seeks to consider the environmental variable during the planning stages. The purpose of this study was to identify the contribution of SEA to support decision-making processes involving the environmental use of transgenic crops in Brazil. Given that this activity is strategic for Brazilian agriculture, we analyzed it according to the parameters that determine good SEA practices. We found out that SEA can contribute to the taking of decisions regarding the commercial use of these cultivars, according to its principles of transparency, social Involvement, environmental planning, broad territorial scope, assessment of cumulative and long-term impacts and continuous environmental monitoring.

Keywords: GMO; biosafety; environmental planning.

\section{Introdução}

A questão dos transgênicos no Brasil é bastante polêmica e divide opiniões entre os diversos setores da sociedade (Guivant, 2006; Veiga, 2007; Camara et al., 2009). No entanto, estima-se, segundo dados da bioindústria, mas sem o respaldo de estatísticas oficiais, que o país é o segundo maior produtor mundial de organismos geneticamente modificados de interesse agrícola, com 40,3 milhões de ha cultivados, sendo precedido pelos EUA e seguido pela Argentina, Índia, Canadá, China, Paraguai e África do Sul (James, 2013).

Pelo fato de os organismos geneticamente modificados (OGMs) possuírem o potencial de ocasionar impactos socioambientais e econômicos, diversas organizações internacionais e nações estabeleceram marcos regulatórios de caráter mandatário ou orientador a seu respeito. Em âmbito global, destaca-se o Protocolo de Cartagena sobre Biossegurança, concebido em 2000 pela Conferência das Partes da Convenção sobre Biodiversidade e que tem por objetivo:

[...] assegurar um nível adequado de proteção no campo da transferência, da manipulação e do uso seguro dos organismos vivos modificados resultantes da biotecnologia moderna que possam ter efeitos adversos na conservação e no uso sustentável da diversidade biológica, levando em conta os riscos para a saúde humana, e enfocando especificamente os movimentos transfronteiriços (Brasil, 2006, art. $1^{\circ}$ ).
O Protocolo tem como premissa a vigência do princípio da precaução nos processos decisórios sobre OGMs, dadas as incertezas científicas sobre os possíveis impactos socioambientais e econômicos advindos de sua utilização, e adota a análise de risco como ferramenta de avaliação caso a caso. O Protocolo se aplica a diversas atividades relativas ao uso de OGMs, tais como seu movimento transfronteiriço, trânsito, manipulação e utilização, sendo utilizado por diversas nações como um modelo orientador na elaboração de normas de biossegurança internas para as pesquisas e comercialização destes organismos. Similarmente, outras organizações internacionais, como a OECD (Organization for Economic Cooperation and Development), FAO (Food and Agriculture Organization of The United Nations), o PNUMA (Programa das Nações Unidas sobre Meio Ambiente), o IPPC (International Plant Protection Convention), a ASEAN (Association of Southeast Asean Nations) e a União Europeia têm adotado a abordagem de avaliação de risco caso a caso para os cultivares geneticamente modificados, como forma de se detectar os possíveis riscos que tais organismos possam acarretar à saúde e ao meio ambiente. O mesmo se sucede para com os demais países que adotaram normatizações sobre biossegurança, como Canadá, Austrália, Nova Zelândia, Argentina, África do Sul, China, Índia, Brasil, dentre outros.

Dentre os riscos ambientais e à saúde advindos da liberação de cultivares transgênicos são considerados, no presente artigo, aqueles provenientes de sua introdução em larga escala e de longo prazo, passíveis de ocasio- 
narem impactos indiretos e cumulativos. Neste sentido, autores como Hindmarsh (1990), Stirling (2000), Whales \& Mythen (2002), Ely (2002), Henry (2006) e Pizella \& Souza (2012a) questionam as atuais abordagens de Análise de Risco e os Estudos de Impactos Ambientais como ferramentas únicas de avaliação de impactos ambientais de OGMs, em razão das dificuldades destes instrumentos em identificar e avaliar os impactos cumulativos, indiretos e de longo prazo resultantes do cultivo de OGMs em larga escala, de liberações coincidentes no espaço envolvendo diferentes tipos de OGMs e dos impactos iniciados após o término dos cultivos, difíceis de serem detectados sem um monitoramento ambiental contínuo.

Linacre et al. (2005) e Pessôa (2007) levantam as oportunidades do uso de ferramentas de análise capazes de lidar com escalas geográficas e temporais mais extensas, com enfoque no planejamento e na participação social, tais como a Avaliação Ambiental Estratégica (AAE), que vem sendo utilizada mundialmente para avaliar os impactos ambientais de Políticas, Planos e Programas (PPPs) propostos pelos governos ou organizações privadas. Em relação aos diversos usos que podem ser feitos da AAE, Therivel (2004) cita propostas de leis, tratados internacionais, políticas econômicas, planos de desenvolvimento, políticas, planos e programas setoriais (tais como nas áreas de transporte, agricultura, resíduos sólidos, energia, saneamento, dentre outras) e políticas, planos e programas territoriais (tais como de bacias hidrográficas, de gestão costeira, manejo florestal, dentre outros).

Diversas nações possuem regulamentações específicas para a AAE, sendo os EUA o primeiro país a mencionar a necessidade de um instrumento que avaliasse as principais políticas governamentais estadunidenses, quando da criação de sua Agência Ambiental, em 1969. Segundo Therivel (2004), até 2003 aproximadamente vinte nações possuíam dispositivos legais de AAE, tendo por base dois modelos emergentes, quais sejam, a Diretiva Europeia 2001/42/EC e o SEA Protocol da Comissão Econômica das Nações Unidas para a Europa (UNECE, na sigla em inglês).

No Brasil, a AAE ainda não se encontra regulamentada, apesar da existência de dois projetos de Lei que se encontram em discussão em instâncias governamentais diferentes: o Projeto de Lei ${ }^{\circ} 2.072$ de 2003, de autoria do deputado Fernando Gabeira, que visa alterar a Lei ${ }^{\circ}$ 6.983, de 31 de agosto de 1981, a fim de dispor sobre a Avaliação Ambiental Estratégica de Políticas, Planos e Programas (Câmara dos Deputados, 2003) e o Projeto de Lei $\mathrm{N}^{\circ} 120$ de 2011, que dispõe sobre o uso da Avaliação Ambiental Estratégica para os órgãos da Administração Pública Direta e Indireta do Estado de São Paulo (Assembleia Legislativa do Estado de São Paulo, 2011). No entanto, há diversos projetos-pilotos realizados no país, com destaque para a AAE do território da Bacia Hidrográfica do Alto Paraguai, realizada pelo Ministério do Meio Ambiente em conjunto com os Estados do Mato Grosso e Mato Grosso do Sul; a AAE da Costa Norte, voltada para o planejamento turístico desta região brasileira; a AAE do Rodoanel de São Paulo (Ministério do Meio Ambiente, 2006), dentre outros.

Ao inserir a questão ambiental nas propostas governamentais, a AAE é um instrumento que possui o potencial de aprimorar a ação estratégica, em função dos benefícios trazidos ao planejamento territorial, por adotar as seguintes premissas (Sadler \& Baxter, 1997; Dalal-Clayton \& Sadler, 2005):

- inserção da sustentabilidade ambiental nos processos decisórios, integrando as diversas dimensões do desenvolvimento (econômica, social, ambiental, política e cultural);

- consideração de alternativas locacionais, tecnológicas e programáticas às PPPs propostas, dificilmente abordadas nas avaliações pontuais de empreendimentos;

- análise de impactos cumulativos, indiretos e de longo prazo, não detectados em atividades pontuais;

- aprimoramento da capacidade institucional na detecção de impactos e de sua consideração nas fases iniciais de planejamento, facilitando a análise dos impactos no âmbito dos projetos;

- melhoria da coordenação entre setores governamentais no processo de elaboração de PPPs;

- auxílio no delineamento das atividades pontuais, em razão da incorporação de objetivos e metas ambientais em políticas, planos e programas que sirvam de base para a elaboração de projetos que considerem de antemão a dimensão ambiental, 
a identificação prévia dos impactos e das informações necessárias para a avaliação de projetos, além da redução do tempo e dos esforços para a realização de revisões;

- engajamento do público interessado e diretamente afetado no processo de elaboração de PPPs.

O Brasil possui um sistema regulatório de OGMs ancorado na Lei de Biossegurança de 2005, que atribui à Comissão Técnica Nacional de Biossegurança (CTNBio) o poder decisório sobre as autorizações de uso destes organismos em suas diversas aplicações, utilizando a análise de risco como instrumento de estudo ambiental para embasar as decisões (Brasil, 2005). No entanto, Pizella \& Souza (2012b), apontam para a insuficiência desta ferramenta de análise para a avaliação dos potenciais impactos econômicos e socioambientais derivados da liberação ambiental em larga escala destes organismos.

Deste modo, tendo em vista que a opção pelo uso da tecnologia transgênica na agricultura é uma questão política com implicações sociais, econômicas e ambientais de longo prazo e considerando a potencialidade do instrumento Avaliação Ambiental Estratégica em avaliar políticas públicas de forma adequada, o presente artigo se propõe a identificar as contribuições da utilização da AAE para subsidiar os processos decisórios que envolvem a liberação ambiental de cultivares transgênicos no Brasil.

\section{Metodologia}

A potencialidade do emprego da Avaliação Ambiental Estratégica no processo decisório envolvendo a liberação de variedades agrícolas geneticamente modificadas será analisada segundo os parâmetros de análise que condicionam suas boas práticas:

- consideração de impactos socioambientais, culturais e econômicos indiretos e cumulativos;

- ampla escala territorial de abrangência;

- interface de ações estratégicas com Políticas, Planos e Programas relacionados;
- consideração de alternativas tecnológicas, locacionais e de desenvolvimento no processo de avaliação;

- possibilidade de participação social nas tomadas de decisão nas etapas iniciais de planejamento da ação estratégica; e

- monitoramento ambiental contínuo e retroativo.

\section{Resultados e discussão}

A liberação ambiental de cultivares transgênicos pode ser considerada uma ação estratégica, pelos seguintes motivos:

- Trata-se de uma questão eminentemente política, não somente técnica (tanto que a instância de tomadas de decisão definitiva sobre a liberação ou não de OGMs é o Conselho Nacional de Biossegurança, formado por 11 Ministros de Estado), pois envolve conflitos de interesses e opções de desenvolvimento;

- Implica um modelo agrícola baseado em monoculturas, influenciando os demais (policulturas, sistemas de cultivo convencional e alternativos). Portanto, relaciona-se também ao planejamento da agricultura;

- Do modo como são autorizadas para plantio, ou seja, para o território nacional como um todo, não se caracterizam como projetos pontuais. Neste sentido, podem-se considerar os processos remetidos à Comissão Técnica Nacional de Biossegurança como Planos ou Programas, que consolidam a Missão ou Política do Ministério da Agricultura, Pecuária e Abastecimento e a Estratégia Nacional de Biotecnologia, ambas tendo por objetivo o estímulo à competitividade do país quanto ao desenvolvimento de cultivares transgênicos;

- Apresenta impactos socioambientais e econômicos potenciais de larga escala, cumulativos e sinérgicos, sejam positivos, negativos ou neutros;

- Relaciona-se com outras estratégias: legislações, políticas e tratados internacionais. 
Como observado por Pizella \& Souza (2012b), os instrumentos Análise de Risco (AR) e Estudos de Impacto Ambiental (EIA), quando utilizados como únicas ferramentas para os estudos ambientais necessários às solicitações de liberação de cultivares transgênicos, não se demonstram adequados, já que:

- tanto a AR quanto o EIA não consideram as Políticas, Planos e Programas que se relacionam a esta forma de tecnologia;

- o Protocolo de Cartagena e a Lei de Biossegurança preveem a realização de análises de risco caso a caso de OGMs, ou seja, que considerem suas características e aquelas do meio onde será introduzida. Como as liberações são realizadas para todo o território nacional, as particularidades de cada local não são consideradas. Tanto a AR quanto o EIA não avaliam ações que ocorrem em larga escala espacial;

- ambos os instrumentos desconsideram a avaliação dos impactos sociais, ambientais e econômicos cumulativos que potencialmente possam surgir com a introdução ambiental de diversos cultivares transgênicos ao longo do tempo;

- o monitoramento ambiental previsto no EIA/ RIMA cessa após o término da atividade, o que impossibilita a avaliação dos impactos negativos previstos e imprevistos que possam ocorrer em um horizonte temporal amplo;

- por não serem instrumentos afeitos ao planejamento ambiental de longo prazo, não possibilitam a participação social efetiva nas tomadas de decisão nas situações que não tratam de atividades pontuais;

- não consideram alternativas de cultivo, tais como o uso de variedades convencionais e/ou orgânicas, por serem instrumentos de avaliação pontual e não de planejamento, que analisam os potenciais impactos sociais, culturais, ambientais e econômicos somente da variedade transgênica.

Segundo Souza (2006), a falta de regulamentação do instrumento da Política Nacional de Meio Ambiente "Sistema Nacional de Informações sobre Meio Ambiente" inviabiliza a eficácia do EIA, por atribuir-lhe funções que não lhe cabem, notadamente a coleta de informações ambientais por parte do proponente. A falta de informações ambientais sistematizadas constitui-se em um exem- plo que dificulta a realização de EIAs no Brasil, assim como a falta de planejamento ambiental. Neste sentido, um instrumento de planejamento que tem o potencial para contribuir para os aspectos acima abordados e que vem sendo utilizado em diversas nações é a Avaliação Ambiental Estratégica, ainda não regulamentada no Brasil, apesar da realização de alguns projetos-pilotos neste sentido, como anteriormente mencionado.

De acordo com a Convenção sobre Diversidade Biológica, a avaliação de impactos ambientais de políticas e programas potencialmente causadores de danos ambientais deve ser utilizada como instrumento decisório, sendo que os países signatários devem aplicar a AAE e o EIA em propostas que impliquem impactos negativos à biodiversidade, de modo a protegê-la e assegurar o uso compartilhado de seus benefícios (International Association for Impact Assessment, 2005).

O planejamento ambiental possibilita uma abordagem transversal dos interesses da coletividade, ou seja, sociais, culturais, ambientais e econômicos, de modo a integrá-los por meio dos instrumentos de política ambiental. A Avaliação Ambiental Estratégica é um instrumento de política ambiental que possibilita a realização de interfaces com diversos objetivos presentes no planejamento territorial. Deste modo, aos objetivos da ação estratégica em questão, no caso, a liberação ambiental de cultivares transgênicos, seriam somados outros objetivos identificados nos planos já elaborados ou previstos para a unidade territorial em estudo.

Dependendo da área geográfica em questão, pode-se efetuar uma AAE considerando os objetivos das legislações, Politicas, Planos e Programas existentes, tais como: PPPs federais e os tratados internacionais, dentre os quais a Constituição Federal de 1988, a Política Nacional do Meio Ambiente (Lei n $\left.{ }^{\circ} 6.938 / 91\right)$, a Política Agrícola, o Sistema Nacional de Unidades de Conservação (Lei no 9.985/00), o Protocolo de Cartagena, a Lei de Biossegurança (Lei n ${ }^{\circ} 11.105 / 05$ ), a Política Nacional de Biotecnologia, Agendas 21 Global, Nacional e Local, a Convenção sobre Diversidade Biológica, a Política Nacional de Biodiversidade, a Lei de Orgânicos (Lei n ${ }^{\circ}$ 10.831/03), o Código de Defesa do Consumidor (Lei $\mathrm{n}^{\circ}$ 8.078/90), as Normativas da CTNBio, a Lei de Proteção de Cultivares (Lei no 9.456/97), o Plano Purianual, os 
Planos de Bacias Hidrográficas, os Planos Diretores municipais, dentre outros.

A interface entre as diversas Políticas, Planos e Programas governamentais é possibilitada pela AAE por meio da análise das compatibilidades entre a ação proposta (seus objetivos e instrumentos para concretizá-los) e outras estratégias de desenvolvimento delineadas para o território em questão, na forma de PPPs (Therivel, 2004). Apesar de a Resolução CONAMA n ${ }^{\circ}$ 01/86 definir que o EIA deve abranger as PPPs relacionadas ao empreendimento, tal premissa não é efetuada eficazmente, já que a atividade, na prática, tem sua localização e tipologia definidas previamente, sem considerar a escolha das opções de desenvolvimento desejadas pela sociedade. A AAE permite que os conflitos existentes devido aos diversos interesses quanto à estratégia em questão sejam clarificados e os critérios sociais, ambientais e econômicos sejam identificados para as tomadas de decisão.

No Brasil observa-se, quando da identificação dos conflitos sobre OGMs, a inexistência de uma Política Nacional de Biossegurança (PNB) que norteie os princípios e procedimentos para a proteção ambiental e da saúde humana e dos demais animais quando da realização de atividades que envolvam a manipulação de seres vivos por meio de engenharia genética. De acordo com Nodari et al. (2002), sua elaboração, no entanto, é fundamental para delinear os rumos da biossegurança e, consequentemente, da biotecnologia no país. Segundo estes autores, dentre os princípios desta Política devem constar a transparência, a precaução, a publicidade e a participação, a fim de proporcionar um maior controle pela sociedade.

Para tais autores, a ausência de uma PNB tem levado à criação, por parte da CTNBio, de uma excessiva quantidade de normativas, muitas das quais dotadas de conteúdo conflituoso ou com pouco detalhamento. Neste sentido, pode-se citar a normativa que dispõe sobre o monitoramento pós-comercial de OGMs, que não apresenta as diretrizes necessárias ao Plano de Monitoramento que necessita ser fornecido pelo proponente. Outro exemplo é a criação recente da normativa sobre os critérios indispensáveis à análise de risco, aprovada somente em 2008, após a liberação comercial de diversas variedades geneticamente modificadas.
A rotulagem seria outro procedimento necessário para possibilitar o desenvolvimento biosseguro destes organismos, a qual se encontra regulamentada, mas desconsiderada nas deliberações da CTNBio. Sua inclusão como um instrumento da PNB poderia torná-la mais eficaz desta forma. Deste modo, a avaliação de uma estratégia de liberação ambiental de determinado cultivar transgênico fica prejudicada sem o estabelecimento de uma PNB, já que os princípios norteadores para tanto não se encontram estabelecidos. Sobrepesa-se que sua inexistência também dificulta uma maior atuação da sociedade sobre as ações dos órgãos integrantes do sistema regulatório de OGMs no país.

Segundo Pizella \& Souza (2012a) há, no Brasil, a predominância de interesses econômicos nas políticas de biotecnologia, em detrimento dos princípios de biossegurança. Como exemplo, pode-se citar documento estratégico do Ministério da Agricultura, Pecuária e Abastecimento, onde se evidencia o direcionamento das políticas governamentais à bioindústria, caracterizando qualquer visão precaucionista quanto à liberação ambiental de OGMs como ideológica e inadequada aos interesses comerciais do país.

Visando ao estímulo ao desenvolvimento da biotecnologia no país, foi elaborada a Estratégia Nacional de Biotecnologia: Política de Desenvolvimento da Bioindústria, firmada como posição oficial do governo na área de biotecnologia e que visa preparar o Brasil para se destacar como um dos líderes no setor dentro de 10 a 15 anos, com a finalidade de:

[...] promover e executar ações com vistas ao estabelecimento de ambiente adequado para o desenvolvimento de produtos e processos biotecnológicos inovadores, estimular o aumento da eficiência da estrutura produtiva nacional, a capacidade de inovação das empresas brasileiras, a absorção de tecnologias, a geração de negócios e a expansão das exportações [...] (MDICE, 2006, p. 9).

Para tanto, a Estratégia focou-se em quatro áreas temáticas, abrangendo saúde humana, agronegócio, saúde animal e indústria, sendo que para cada área foram definidos diretrizes, objetivos e custos estimados, considerando-se três áreas de análise: alvos estratégicos, definidos no âmbito empresarial como alvos com grande 
potencial de mercado a curto e médio prazos, tendo em vista o aumento da competitividade brasileira na área de biotecnologia; áreas priorizadas, constituindo-se em produtos de relevância para o atendimento das demandas sociais e com potencial de mercado significativo; e áreas de fronteira, inovações tecnológicas de alto valor agregado com potencial para novos mercados (MDICE, 2006).

Com o intuito de criar um ambiente adequado ao atendimento das necessidades da bioindústria brasileira, foram elaboradas ações estruturantes abrangendo sistemas de financiamento, preparação de recursos humanos e infraestrutura, além da consolidação de marcos regulatórios que se adéquem às finalidades pretendidas na Estratégia. No âmbito do financiamento, abordou-se a necessidade da criação de uma instância executiva no Comitê Nacional de Biotecnologia que vise ao desenvolvimento de programas que atendam aos objetivos e metas de investimentos pretendidos.

Como objetivo geral, destaca-se a promoção de ações de fomento para a formação de empresas ou rede de empresas inovadoras na área de biotecnologia, utilizando-se, dentre outras ferramentas, de desoneração tributária para a modernização industrial e a exportação de produtos biotecnológicos (MDICE, 2006). No entanto, a elaboração da Estratégia Nacional de Biotecnologia previamente à Política Nacional de Biossegurança pode dificultar que outras preocupações da sociedade, como a utilização segura destes produtos, sejam incorporadas na concretização desta estratégia. A elaboração da PNB, deste modo, seria um importante subsídio para a realização de uma AAE relacionada à liberação ambiental de cultivares transgênicos como uma das Políticas a lhe fazerem interface.

A AAE contempla uma fase inicial denominada escopo, que delimita os procedimentos da AAE, identificando as etapas decisórias da ação estratégica, de modo que haja uma integração entre ambas, além de serem identificados os atores sociais que irão participar de seu processo. Para Therivel (2004), ao menos três grupos devem estar envolvidos em qualquer AAE: os tomadores de decisão e seus consultores, as organizações ambientais e o público interessado. Quanto a quem deve conduzir ou elaborar a AAE, a autora sugere um trabalho conjunto entre especialistas e autoridades governamentais, diferentemente do EIA, que é realizado apenas por con- sultores. Há a identificação de outras ações estratégicas relacionadas à PPP em questão, a fim de conciliar seus objetivos e metas, evitando conflitos entre as propostas.

$\mathrm{Na}$ ausência de uma avaliação estratégica, o processo atual que culmina na liberação de cultivares transgênicos desconsidera outras PPPs que poderiam influenciar a tomada de decisões, conduzindo a conflitos potenciais relacionados às opções de desenvolvimento que são socialmente desejadas para uma determinada localidade. Caso a AAE fosse implementada, outras Políticas, Planos e Programas seriam identificadas nas regiões brasileiras onde os cultivares seriam liberados (ou mesmo para o país como um todo) e uma análise de compatibilidade entre objetivos apresentaria os diversos interesses existentes, que poderiam condicionar a tomada de decisão no sentido favorável, contrário ou com condicionantes. A Figura 1 ilustra algumas das potenciais PPPs que seriam identificadas neste momento da AAE. A Política Nacional de Biossegurança está apresentada em tracejado devido a sua inexistência até o momento.

Depois de realizadas as interfaces com outras PPPs, a próxima etapa da AAE é a elaboração de um estudo de base ou diagnóstico ambiental da área em estudo, onde são levantadas informações ambientais relevantes que demonstram a situação atual do meio ambiente para posterior identificação e avaliação dos potenciais impactos das medidas de ação. Therivel define a base ambiental como "[...] o estado atual do ambiente e seu provável futuro na ausência da ação estratégica" (Therivel, 2004, p. 92). No caso de pedido de liberação ambiental de cultivares transgênicos, os temas e critérios ambientais para a elaboração do diagnóstico na área de abrangência, que pode ser um bioma, estado ou região, podem ser identificados a partir dos termos de referência do EIA, conforme presentes na Resolução CONAMA $n^{\circ} 305 / 02$ (Brasil, 2002), que define o procedimento para o licenciamento ambiental de transgênicos no Brasil. O diagnóstico ambiental se configura, portanto, em uma importante etapa para levantar os potenciais impactos que serão desencadeados com a liberação de uma cultivar transgênica. Deste modo, alternativas locacionais, tecnológicas e de desenvolvimento para a liberação podem ser levantadas, tendo em vista recortes territoriais amplos, uma atribuição que não cabe ao EIA ou à Análise de Risco. 


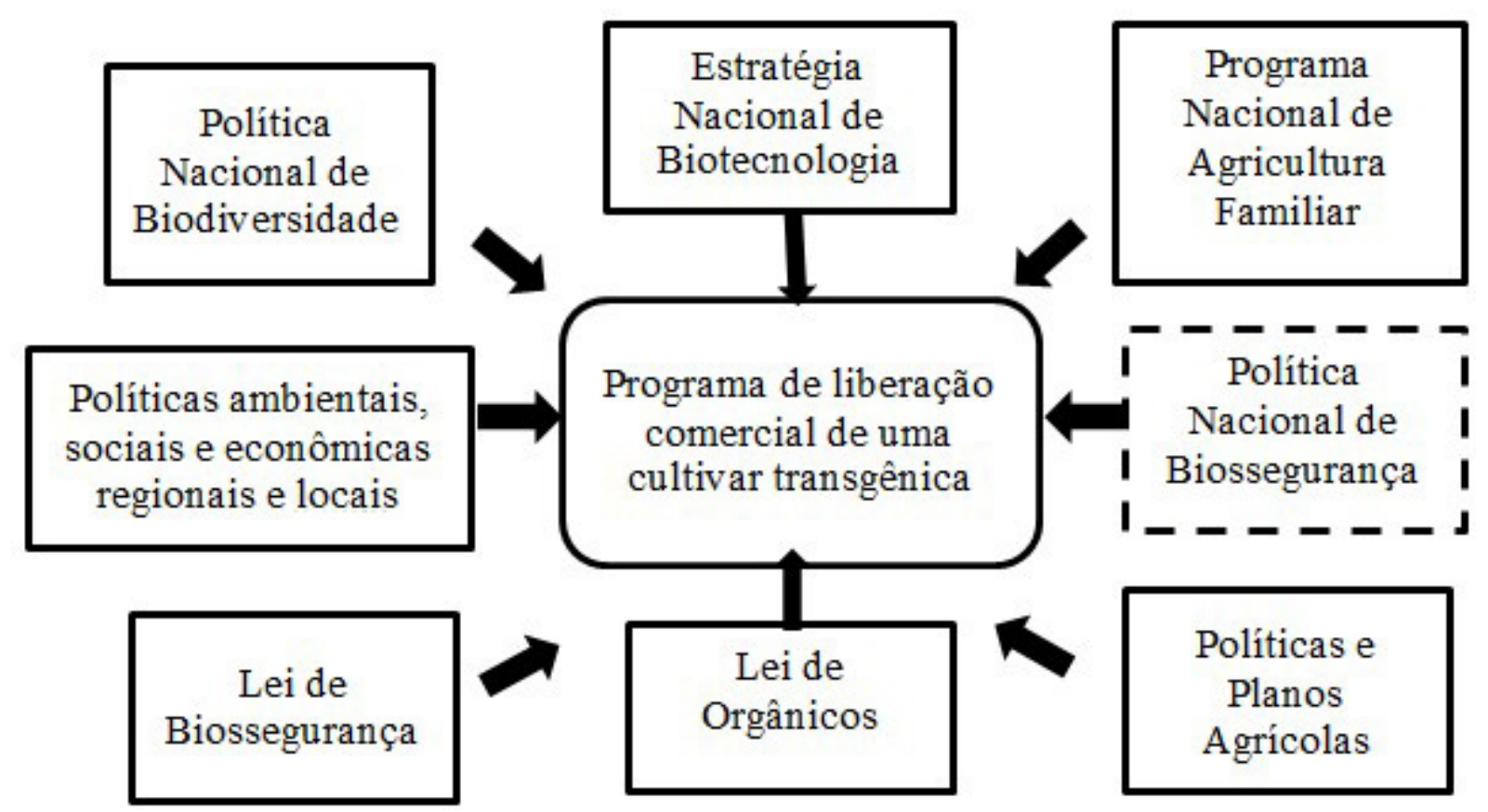

FIGURA 1 - Políticas, Planos e Programas - PPPs que fazem interface com a estratégia de análise de pedido de liberação ambiental de cultivares transgênicos, na fase de escopo da Avaliação Ambiental Estratégica - AAE.

FONTE: Elaboração dos autores.

A necessidade de levantar e analisar as alternativas tecnológicas e de desenvolvimento relacionadas à ação estratégia em estudo se dá em razão de o uso de OGMs na agricultura ser uma opção de desenvolvimento, que necessita ser avaliada perante outras em seus riscos e benefícios, sendo que a decisão final precisa atender aos anseios da sociedade sob o prisma do desenvolvimento sustentável. A aplicação da AAE é uma ferramenta que possibilita a tomada de decisão sob esta ótica e, portanto, permite um maior controle social por minimizar a predominância de interesses setorizados. A elaboração de alternativas locacionais e de desenvolvimento possibilita, por meio de cenários ambientais envolvendo a análise e a predição de impactos, um embasamento científico abrangente para a escolha da melhor alternativa apresentada (Partidário, 2002).

AAAE é fundamental para subsidiar as tomadas de decisão nos processos iniciais de planejamento, fornecendo uma visão ampla dos impactos indiretos e cumu- lativos das alternativas propostas, que não são passíveis de serem analisados por EIAs, que tratam de atividades pontuais e não de larga escala (Therivel, 2004). De acordo com Souza (2006), os estudos efetuados para o diagnóstico ambiental, na medida em que possibilitam a sistematização de informações ambientais em termos geográficos, auxiliam tanto o planejamento territorial quanto a realização de EIAs. Dentre as contribuições destes estudos, situam-se as análises de alternativas locacionais, delimitação da área de influência e definição dos termos de referência para empreendimentos.

Por meio da existência de informações ambientais sistematizadas na etapa diagnóstica, é possível estabelecer, com a participação de uma equipe técnica multidisciplinar, de órgãos governamentais e de organizações da sociedade civil interessadas, as áreas mais frágeis e mais adequadas ao cultivo de OGMs.

No entanto, para a realização desta etapa, necessita-se de informações ambientais muitas vezes inexistentes 
nos órgãos governamentais, apesar de a Política Nacional de Meio Ambiente atribuir ao Poder Público sua coleta e disponibilização ao instituir o sistema de informações ambientais como um de seus instrumentos. Na ausência de dados, o processo inicial para a elaboração destes estudos é, deste modo, dificultada. Entretanto, assim como para o EIA, apenas as informações necessárias para a avaliação devem ser utilizadas, evitando a perda de tempo e recursos que poderiam dificultar sobremaneira a AAE. Dentre as informações necessárias para a realização de um diagnóstico ambiental que subsidie uma AAE para cultivares transgênicos, podem-se exemplificar:

- existência de modalidades de Unidades de Conservação e áreas especialmente protegidas, com especial atenção àquelas que possuam espécies-alvo que possam ser impactadas negativamente pela introdução do OGM em questão;

- existência de cultivares locais ou crioulas a serem protegidas de possíveis contaminações por material transgênico, tais como as culturas de milho, feijão, etc., e sistemas convencionais ou orgânicos de produção;

- identificação das espécies-chave, processos ecológicos e as interações para o funcionamento do ecossistema (ou ecossistemas) da área de abrangência da AAE. Nestes, a identificação das potenciais espécies-alvo, processos ecológicos e interações que possam ser afetados direta ou indiretamente pela introdução do OGM e seu agroquímico associado, no caso de cultivares resistentes a herbicidas ou produtoras de inseticidas;

- os padrões de qualidade ambiental para a água e o solo;

- as terras indígenas, quilombolas e outras áreas de restrição.

As ferramentas para a avaliação de impactos ambientais em AAE dependem do objeto em avaliação, ou seja, se se trata de uma Política, Plano ou Programa e do recorte territorial envolvido (Partidário, 2002). Em casos de grandes incertezas quanto aos potenciais impactos, como a liberação de cultivares transgênicos, podem ser empregadas como metodologias de avaliação de impactos a cenarização ambiental, análise multicriterial, checklists, redes de interação e a análise de risco (Stirling, 2000; Henry, 2006). Deste modo, pode-se inferir que a AR é uma ferramenta metodológica utilizada para a predição de impactos ambientais tanto em AAE quanto em EIA.

Além do diálogo com outras PPPs, da elaboração do diagnóstico ambiental, do levantamento e da avaliação dos impactos de alternativas tecnológicas, locacionais e desenvolvimento, uma das premissas da AAE é sua natureza participativa, possibilitando que o julgamento sobre o problema em questão não permaneça em mãos de determinados setores da sociedade, excluindo os demais agentes afetados pelas tomadas de decisão. Além disto, formas de negociação podem ser propiciadas, de modo a se criar um consenso que implique a minimização de conflitos, já que os diversos pontos de vista podem ser considerados (Therivel, 2004).

A elaboração de um planejamento setorial de longo prazo em um Estado democrático necessita de uma ampla participação social prévia e processual, abrangendo os setores interessados e afetados. As incertezas científicas sobre as consequências da liberação no meio ambiente de cultivares transgênicos tanto para os ecossistemas quanto para a saúde humana e para as cadeias produtivas requerem análises ambientais que abranjam não apenas conteúdos técnicos, mas também questões de caráter ético que reflitam a posição dos grupos sociais envolvidos. Neste sentido, os estudos ambientais necessários para as deliberações sobre a comercialização destes cultivares necessitam da constituição de fóruns de debate com representantes da sociedade e dos governos federal, estadual e municipal, permitindo uma maior transparência no processo. Sendo uma fase prévia ao processo, possibilita o envolvimento do público interessado na elaboração da ação estratégica, garantindo uma maior transparência.

No caso em questão, é possível identificar os possíveis agentes sociais de uma AAE por meio da observação dos participantes das audiências públicas até então realizadas para alguns cultivares transgênicos e por meio da literatura sobre o tema, quais sejam: órgãos governamentais responsáveis pela regulação de OGMs; especialistas em biossegurança, ecologia, meio ambiente, direito, sociologia, agronomia, engenharia florestal; produtores rurais, entomologistas, nutricionistas, médicos, bioquímicos, organizações da sociedade civil, dentre outros. 
Caso haja uma decisão favorável à liberação de determinado cultivar transgênico em uma unidade territorial específica, uma vantagem do uso da AAE é possibilitar um monitoramento pós-comercial contínuo que vise à retroalimentação da decisão tomada. O monitoramento é fundamental para a detecção dos impactos potenciais previstos nas fases de escopo e avaliação (com determinação de seu grau de amplitude e magnitude) da AAE, assim como aqueles imprevistos. Desta forma, podem-se adotar medidas mitigadoras, preventivas e mesmo a reavaliação da escolha tomada.

O monitoramento requer metodologias que promovam a integração entre os aspectos espaciais e temporais dos ecossistemas ou de outro recorte territorial abordado. Para Graef et al. (2004), a unidade territorial propícia para efetuar o monitoramento nestas situações é a ecorregião, em função da existência de uma certa homogeneidade florística e faunística em sua composição. Tais autores salientam as dificuldades de identificação dos riscos e benefícios potenciais da liberação ambiental de cultivares transgênicos devido à complexidade dos ecossistemas, sendo que os impactos ambientais variam espacial e temporalmente de acordo com as características agroambientais, do cultivar e da sua modificação genética, além das práticas agrícolas adotadas. Neste sentido, enfatizam a necessidade de informações sobre os fatores ambientais escolhidos e a utilização de sistemas de georreferenciamento para identificar os locais mais adequados para a delimitação de sistemas de monitoramento ambiental.

A implementação da AAE no planejamento ambiental pode trazer contribuições nas tomadas de decisão referentes às ações estratégicas em questão devido a sua característica em lidar com amplas áreas geográficas. Já o EIA, apesar das atribuições de considerar as áreas de influência dos empreendimentos avaliados e do monitoramento ao longo do ciclo de vida da atividade, dificilmente cumpre seu papel sem a prévia existência de avaliações de áreas geográficas amplas. Desta forma, a AAE é uma ferramenta aplicável para o monitoramento ambiental, especialmente em casos de imprevisibilidade dos impactos, uma característica intrínseca aos casos em questão.

Pode-se ilustrar as contribuições da AAE para a tomada de decisão sobre a liberação de cultivares transgênicos considerando um Programa hipotético de liberação em um Estado ou região, conforme apresentado na Figura 2.

Deste modo, pode-se concluir que Avaliação Ambiental Estratégica traria contribuições significativas ao processo decisório envolvendo a liberação ambiental de cultivares transgênicos, já que, dados os seus princípios e procedimentos, é um instrumento que permite um melhor balanceamento entre as diversas visões sociais a respeito do assunto, além de considerar aspectos legais e de planejamento ambiental de longo prazo.

Considerando que esta ação estratégica pode se sujeitar à realização de uma AAE, o Estudo de Impacto Ambiental pode ser aplicado para a avaliação de atividades pontuais com cultivares transgênicos, como sua liberação ou não em um agroecossistema. A análise de risco, por sua vez, pode ser utilizada como uma ferramenta que subsidie a avaliação de impactos, como observado na Resolução CONAMA no 305/02. No entanto, é importante salientar que a AAE e o EIA são instrumentos de AIA que se complementam, pois as informações mais generalizadas, de conteúdo estratégico da AAE, subsidiam a elaboração dos termos de referência do EIA e os resultados destes, obtidos por observações locais mais detalhadas, podem levar a readequações da ação estratégica. AAnálise de Risco, por sua vez, é uma importante ferramenta para fornecer critérios a serem ponderados nos estudos ambientais, tanto para a criação de parte dos termos de referência do EIA quanto para a identificação de critérios ambientais utilizados na etapa de diagnóstico ambiental da AAE.

\section{Conclusões}

A questão dos transgênicos, sobretudo os destinados ao uso agrícola, levanta diversas polêmicas em termos mundiais, devido ao elevado grau de incertezas quanto aos potenciais impactos ambientais e socioeconômicos decorrentes de sua liberação em larga escala. Neste sentido, as opiniões se dividem em torno da segurança destes organismos, partindo de premissas distintas, tais como o Princípio da Equivalência Substancial, que determina a similaridade entre os OGMs e as plantas convencionais, e o Princípio da Precaução, que 


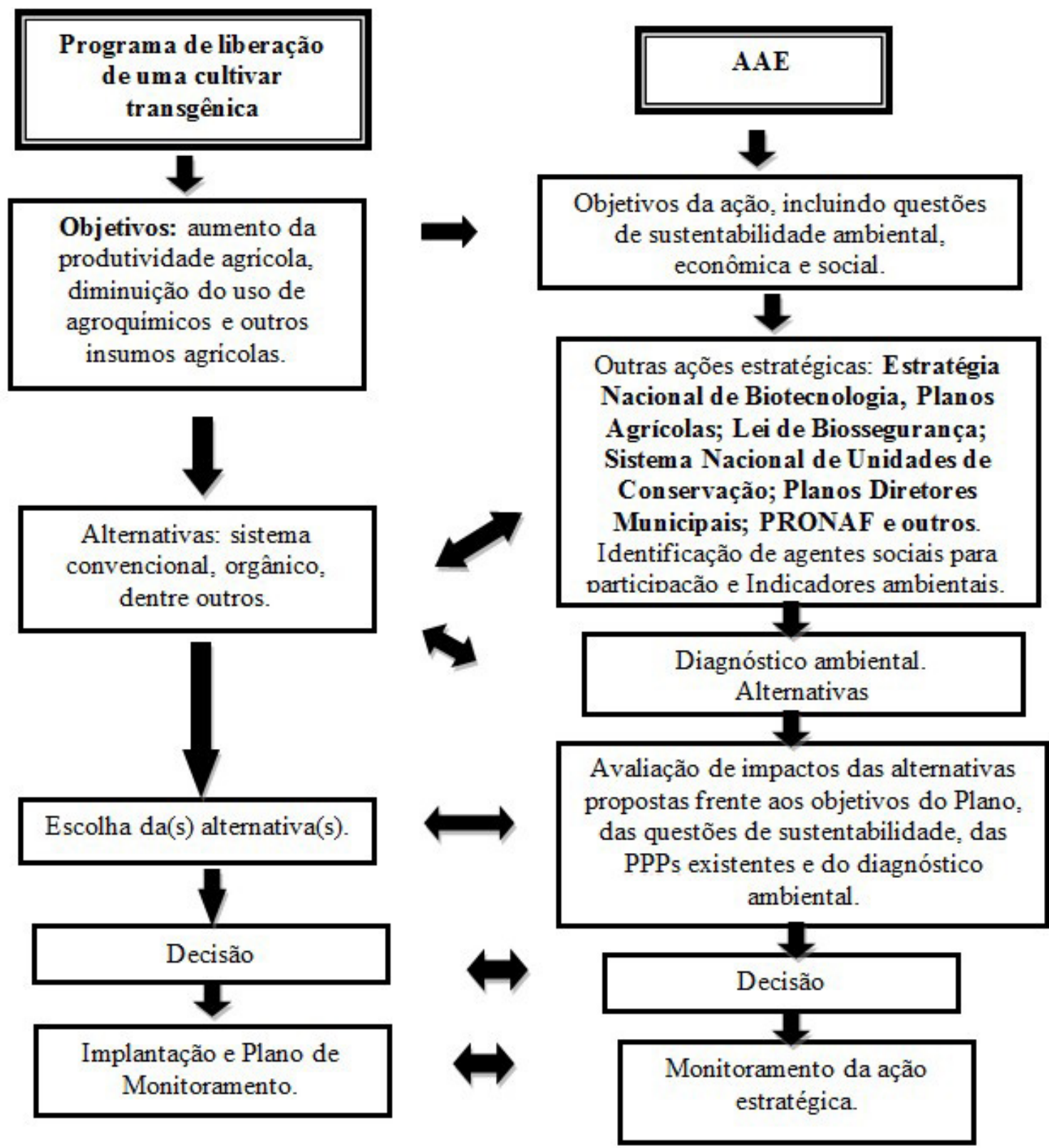

FIGURA 2 - Exemplo de uma ação estratégica envolvendo a análise para a tomada de decisão acerca da liberação ambiental ou não de um cultivar transgênico e as contribuições da Avaliação Ambiental Estratégica - AAE.

FONTE: Modificado de Therivel (2004). 
considera as incertezas quanto aos impactos ambientais destes organismos.

O objetivo deste artigo consistiu em analisar as possíveis contribuições da Avaliação Ambiental Estratégica para a tomada de decisões sobre a liberação ambiental ou não de cultivares transgênicos, tendo em vista os potenciais impactos socioambientais e econômicos que esta opção tecnológica pode ocasionar em larga escala e em longo prazo no território brasileiro.

No Brasil, o único instrumento de estudo ambiental adotado nos processos decisórios envolvendo OGMs é a análise de risco, a qual, como observado, não leva em conta diversos critérios fundamentais para a identificação e avaliação de seus impactos ambientais potenciais, tanto positivos quanto negativos, tais como: os impactos cumulativos, indiretos e cumulativos; as alternativas tecnológicas, principalmente quanto a outros sistemas de cultivo; as alternativas locacionais, já que as análises não consideram as particularidades ambientais, sociais e econômicas brasileiras; a compatibilidade com as leis, Políticas, Planos e Programas relacionados e o Plano de Monitoramento pós-comercial.

O instrumento EIA/RIMA, do mesmo modo, não se mostra adequado para a avaliação destes cultivares, devido ao seu caráter pontual. Deste modo, o EIA não tem a atribuição de considerar impactos indiretos, cumulativos e de longo prazo, assim como as alternativas tecnológicas e locacionais aos empreendimentos sujeitos à sua avaliação.

Ao analisar o instrumento Avaliação Ambiental Estratégica, algumas de suas características mostraram-se adequadas para a avaliação dos impactos ambientais de cultivares transgênicos a serem liberados em larga escala, tais como:

- Tais liberações não se caracterizam como atividades pontuais, pois, uma vez autorizado o plantio, o mesmo se dá em todo o território nacional. Neste sentido, constituem-se em uma ação estratégica compatível com Planos e Programas, com potencial de ocasionar impactos ambientais em larga escala, cumulativos, indiretos e de longo prazo, tanto ambientais como sociais e econômicos. Além disto, fazem parte de uma política governamental de desenvolvimento do setor agrícola, como explicitado na Estratégia Nacional de Biotecnolo- gia e na missão do MAPA, que se relaciona com outras Leis e PPPs. Portanto, estas devem ser consideradas em qualquer processo de tomada de decisões. A AAE, sendo um instrumento de planejamento ambiental, possui como atribuição a avaliação de impactos ambientais, sociais e econômicos de ações estratégicas e, portanto, tem a potencialidade de servir como instrumento de AIA nestes casos;

- A AAE considera as alternativas de localização para a implementação de PPPs e outras ações estratégicas. Se as informações ambientais encontrarem-se sistematizadas e, de preferência, georreferenciadas, é possível tomar decisões de teor preventivo ou precaucionista sobre a liberação ambiental de cultivares transgênicos, definindo-se os locais mais adequados para tanto ou sua não liberação, dependendo da vulnerabilidade do meio.

- As alternativas tecnológicas também são contempladas na AAE, especialmente em se tratando de estratégias de desenvolvimento, como no presente caso. De acordo com outras Políticas, Planos e Programas correlatos, assim como por meio da participação pública, é possível que a tomada de decisões sobre a liberação ou não de um cultivar transgênico em um determinado recorte territorial seja realizada de forma legítima e respeitando os condicionantes postos em lei. A escolha sobre os rumos da agricultura pode ser feita deste modo, considerando um horizonte de longo prazo.

- A participação social é crucial para a realização de uma AAE, tal como presente em suas boas práticas e na Diretiva Europeia sobre AAE e no SEA Protocol, os guias mais utilizados para a criação de legislações sobre AAE no mundo. No entanto, mais estudos relacionados às formas de participação direta da sociedade organizada precisam de atenção.

- A rotina de monitoramento da ação estratégica é condicionante para uma boa prática de $\mathrm{AAE}$, trazendo contribuições significativas para o tratamento do caso em questão, em função das incertezas quanto aos impactos de longo prazo decorrentes do plantio em larga escala destes cultivares. Deste modo, medidas de emergência em caso de impactos significativos podem ser acionadas, de mitigação, prevenção e precaução, na medida em que novos conhecimentos vão sendo adquiridos. A revisão da ação estratégica também pode ser realizada em função do monitoramento. 
Deste modo, pode-se concluir que a AAE consiste em um instrumento de AIA adequado para a tomada de decisões sobre a liberação ambiental de cultivares transgênicos no Brasil.

\section{Referências}

Assembleia Legislativa do Estado de São Paulo. Projeto de Lei $n^{\circ} 120$, de 2011. Dispõe sobre a obrigatoriedade da Avaliação Ambiental Estratégica (AAE) para os órgãos da Administração Pública Direta e Indireta do Estado de São Paulo. São Paulo: Diário Oficial Estado de São Paulo de 19/03/2011. Disponível em: $<$ http://www.al.sp.gov.br/propositura/?id=1000423 $>$.

Brasil. Resolução CONAMA no 305, de 12 de junho de 2002. Dispõe sobre Licenciamento Ambiental, Estudo de Impacto Ambiental e Relatório de Impacto no Meio Ambiente de atividades e empreendimentos com Organismos Geneticamente Modificados e seus derivados. Brasília: DOU de 4/07/2002.

Brasil. Lei $n^{\circ} 11.105$, de 24 de março de 2005. Regulamenta os incisos II, IV e V do $\S 1^{\circ}$ do art. 225 da Constituição Federal, estabelece normas de segurança e mecanismos de fiscalização de atividades que envolvam organismos geneticamente modificados - OGM - e seus derivados, cria o Conselho Nacional de Biossegurança - CNBS, reestrutura a Comissão Técnica Nacional de Biossegurança-CTNBio, dispõe sobre a Política Nacional de Biossegurança - PNB, revoga a Lei no 8.974, de 5 de janeiro de 1995, e a Medida Provisória n ${ }^{\circ}$ 2.191-9, de 23 de agosto de 2001 , e os arts. $5^{\circ}, 6^{\circ}, 7^{\circ}, 8^{\circ}, 9^{\circ}, 10$ e 16 da Lei ${ }^{\circ}$ 10.814 , de 15 de dezembro de 2003, e dá outras providências. Brasília: DOU de 28/03/2005.

Brasil. Decreto $n^{\circ} 5.705$, de 16 de fevereiro de 2006. Promulga o Protocolo de Cartagena sobre Biossegurança da Convenção sobre Diversidade Biológica. Brasília: DOU de 16/02/2006.

Camara, M. C.; Marinho, C. L. C.; Guilam, M. C. R.; Nodari, R. O. Transgênicos: avaliação da possível (in)segurança alimentar através da produção científica. História, Ciências, Saúde-Manguinhos, 16(3), 669-681, 2009.

Câmara dos Deputados. Projeto de Lei $n^{\circ}$ 2.072, de 2003. Altera a Lei $\mathrm{n}^{\circ} 6.938$, de 31 de agosto de 1981, a fim de dispor sobre a avaliação ambiental estratégica de políticas, planos e programas. Disponível em: <http://www.camara.gov.br/ proposicoesWeb/prop_mostrarintegra;jsessionid $=$ ECDE41E 2246D0DE627330F5CE4371CA9.proposicoesWeb1?codteo $\mathrm{r}=166730$ \&filename $=\mathrm{PL}+2072 / 2003>$.

\section{Agradecimentos}

Agradecemos à CAPES (Coordenação de Aperfeiçoamento de Pessoal de Nível Superior) pelo financiamento concedido.

Dalal-Clayton, B.; Sadler, B. Strategic Environmental Assessment: A Sourcebook and Reference Guide to International Experience. London: Earthscan, 2005.

Ely, A. Globalization and International Governance of Modern Biotechnology: Evaluating Environmental Risks of Bt Mayze in the US and EU: Lessons and Challenges for Kenya. In: Workshop on Globalization and Governance of Modern Biotechnology, with special reference to Kenya. Nairóbi, 28 de nov. 2002. Disponível em: <http://r4d.dfid.gov.uk/PDF/ Outputs/Mis_SPC/R7626-ely_maize_2002.pdf>.

Graef, F.; Schmidt, G.; Schröder, W.; Stachow, U. Determining ecoregions for environmental and GMO monitoring networks. Environmental Monitoring and Assessment, 108, 189-2003, 2004.

Guivant, J. S. Transgênicos e percepção pública da ciência no Brasil. Ambiente e Sociedade, 9(1), 1-30, 2006.

Henry, C. Cumulative long-term effects of genetically modified (GM) crops on human/animal health and the environment: risk assessment methodology. UK: Central Science Laboratory, 2006. 236 p. Relatório Final, no 07-0402/2005/414455/Mar/B4.

Hindmarsh, R. The need for effective assessment: sustainable development and the social impacts of biotechnology in the third world. Environmental Impact Assessment Review, 10, 195-208, 1990.

International Association for Impact Assessment. Biodiversity in Impact Assessment. Special Publication Series, 3, 1-4, 2005.

James, C. Global Status of Commercialized Biotech/GM Crops: 2013. ISAAA Brief 46, 2013. Disponível em: <http:// www.isaaa.org>.

Linacre, N. A.; Gaskell, J.; Rosegrant, M. W.; Falk-Zepeda, J.; Quemada, H.; Halsey, M.; Birner, R. Strategic Environmental Assessment: Assessing the Environmental Impact of Biotecnology. Washington: International Food Policy Research Institute, 2005. 
MDICE - Ministério do Desenvolvimento, Indústria e Comércio Exterior. Estratégia Nacional de Biotecnologia: Política de Desenvolvimento da Bioindústria. Brasília: Fórum de Competitividade de Biotecnologia, 2006.

Ministério do Meio Ambiente. Seminário Latino-Americano sobre AAE-principais conclusões. Relatório, 11 de setembro de 2006. Disponível em: <http://www.mma.gov.br/estruturas/ sqa_pnla/_arquivos/relatorio.pdf $>$.

Nodari, R.; Guerra, M. P.; Valle, S. Política Nacional de Biossegurança. Jus Navigandi, 6(56), 2002. Disponível em: <http:// jus2.uol.com.br/doutrina/texto.asp?id=2880>.

Partidário, M. R. Avaliação Ambiental Estratégica. Brasília: MMA/SQA, 2002.

Pessôa, L. T. G. Metodologia de Avaliação Ambiental Estratégica de Organismo Geneticamente Modificado na Agricultura. Rio de Janeiro, Tese (Doutorado em Ciências) - UFRJ, 2007.

Pizella, D. G.; Souza, M. P. Análise dos aspectos institucionais da regulação de OGMs no Brasil: boas práticas de governança ambiental? Desenvolvimento e Meio Ambiente, 25, 27-37, $2012 \mathrm{a}$.
Pizella, D. G.; Souza, M. P. Brazilian GMO Regulation: does it have an environmental approach? Journal of Environmental Assessment Policy and Management, 14(2), 1-16, $2012 \mathrm{~b}$.

Sadler, B.; Baxter, M. Taking Stock of SEA. Environmental Assessment, 5(1), 14-16, 1997.

Souza, M. P. A base de referência e a avaliação de impacto ambiental. Anais $1^{\circ}$ Simpósio de Geotecnologias no Pantanal, Campo Grande, Brasil, 11-15 de novembro de 2006, Embrapa Informática Agropecuária/INPE, p. 749-756, 2006.

Stirling, A. A precautionary approach to technological appraisal? A multicriteria mapping of genetic modification in UK agriculture. TA-Datenbank Nachrichten, 3(9), 39-51, 2000.

Therivel, R. Strategic Environmental Assessment in Action. London: Earthscan Publications Limited, 2004.

Veiga, J. E. Introdução. In: Veiga, J. E. (Org.). Transgênicos: sementes da discórdia. São Paulo: SENAC, p. 9-23, 2007.

Wales, C.; Mythen, G. Risky Discourses: The Politics of GM Foods. Environmental Politics, 11(2), 121-144, 2002. 